\title{
The role of a reconstruction subspecialty group in the national surgical specialty association: the journey, experiences and output for the advancement of head and neck reconstruction
}

\author{
Michael W. S. $\mathrm{Ho}^{1 \wedge}$, Michael Nugent ${ }^{2}$ \\ ${ }^{1}$ Oral and Maxillofacial Surgery, Leeds Dental Institute, Leeds, UK; ${ }^{2}$ Oral and Maxillofacial Surgery, Sunderland Royal Hospital, Sunderland, UK \\ Contributions: (I) Conception and design: Both authors; (II) Administrative support: Both authors; (III) Provision of study materials or patients: \\ Both authors; (IV) Collection and assembly of data: Both authors; (V) Data analysis and interpretation: Both authors; (VI) Manuscript writing: Both \\ authors; (VII) Final approval of manuscript: Both authors. \\ Correspondence to: Michael W. S. Ho. Consultant Oral and Maxillofacial Surgeon, Oral and Maxillofacial Surgery, Leeds Dental Institute, Clarendon \\ Way, LS2 9LU, Leeds, UK. Email: m.ho@leeds.ac.uk.
}

\begin{abstract}
The British Association of Oral and Maxillofacial Surgeons (BAOMS) developed the roles of Sub-Specialty Interest Group (SSIG) lead and deputy leads within the specialty in 2013. This article details the range of activities engaged within the specified duration and discusses the impact that the SSIG has had on the specialty association, membership and how these roles can enrich the experience of the membership and SSIG lead and/or deputy lead. Overall, the role of the Reconstruction SSIG has been pivotal in tackling important and topical issues in head and neck reconstruction in a timely manner for the specialty. The concept of a SSIG has been a successful innovation by BAOMS and the authors commend the BAOMS council for this idea. The SSIG has demonstrated engagement in clinical effectiveness and quality improvements activities in addition to the following role(s): promotion and organisation of simulated educational events, encourage constructive, structured debate within the specialty around specific topical issues in head and neck reconstruction and engagement of membership to survey contemporary practice logistics. The SSIG has contributed significantly to the development of service evaluation registry and/ or audit development in the BAOMS Quality and Outcomes in Oral and Maxillofacial Surgery (QOMS), quality improvement initiative. We would suggest that surgical specialty associations consider this model to enable subspecialties to develop and flourish, with the support of the respective council boards.
\end{abstract}

Keywords: Reconstruction; head and neck; specialty development; quality improvement

Received: 01 January 2021; Accepted: 24 December 2021; Published: 10 March 2022.

doi: $10.21037 /$ fomm-21-3

View this article at: https://dx.doi.org/10.21037/fomm-21-3

\section{Introduction}

The British Association of Oral and Maxillofacial Surgeons (BAOMS) developed the roles of Sub-Specialty Interest Group (SSIG) lead and deputy leads within the specialty in 2013. the subspecialties included in this initiative are aesthetics, cleft, craniofacial, deformity, oral and dentoalveolar surgery, oncology, paediatric, reconstruction, salivary, skin surgery, temporomandibular joint surgery and trauma. The SSIG lead and deputy lead has responsibility to:

(I) BAOMS council:

(i) To be a point of contact for members of their group;

(ii) To be a point of contact for BAOMS council to

\footnotetext{
^ ORCID: 0000-0001-9810-3136.
} 
that group;

(iii) May be asked to represent BAOMS at meetings relevant to their SSIG;

(II) Initiate constructive engagement with their SSIGmain role is to engage with their SSIG;

(III) Develop the SSIG element of the BAOMS website with Web Editor:

(i) General discussion forum moderator;

(ii) Case discussion forum;

(iii) Professional information and links;

(iv) Patient information and links;

(IV) Engage in clinical effectiveness programme:

(i) Attend meetings of Clinical Effectiveness Committee (CEC) (usually 3 days per year);

(ii) Audits and guidelines-update/develop national audits for revalidation and guidelines;

(iii) Patient information leaflets-maintain and update;

(iv) Commissioning of services related to the SSIG:

* Collect and collate information within their SSIG;

* Represent BAOMS, when asked, on and regional bodies in their SSIG;

(V) Respond to engagement with the public, professional and media communications sub-committee;

(i) News monitoring;

(ii) Engage with any public relations campaigns;

(VI) Support continuing professional development and education-collate/generate/publicise resources especially the BAOMS website;

(VII) Engage with the consultant and specialists groupprovide support and advice for colleagues;

(VIII) Promote research within each respective SSIGwork with BAOMS research lead and National Facial, Oral and Oculoplastic Research Centre (NFORC)/Saving Faces.

Performance to be reviewed annually by the chair of BAOMS council against this list of roles and responsibilities. Where a lead and/or deputy lead fails to engage with BAOMS council or members of the group, they will be requested to demit office.

The authors were lead $(\mathrm{MH})$ and deputy lead $(\mathrm{MN})$ for the BAOMS Reconstruction SSIG from November 2015 to July 2020. This article details the range of activities engaged within the specified duration and discusses the impact that the SSIG has on the specialty association, membership and how these roles can enrich the experience of the SSIG lead/ deputy lead.

\section{Activities of the BAOMS Reconstruction SSIG [2016-2020]}

\section{6}

This was the first calendar year of activity by the authors jointly within the SSIG. One of the first commitments was engagement with the CEC of BAOMS. The issue of outcome of reconstructive surgery and availability of data at the unit level was raised and engagement with the BAOMS endorsed UK National Flap Register [UKNFR developed by the British Association of Plastic and Reconstructive Surgeons collaboratively (but not exclusively) with input from BAOMS fellows], was to be evaluated. The main objective for 2016 therefore was to increase membership uptake on the use of the UKNFR. Informal feedback from colleagues in the UK seemed to indicate that the amount of data required has made contribution to the database less popular. In view of this a national survey was conducted to evaluate the practice of data collection for reconstructive surgical outcomes amongst UK Oral and Maxillofacial Surgeons (OMFS). An online SurveyMonkey questionnaire was posted on the Reconstruction SSIG web discussion forum hosted within the BAOMS website. The questions and responses have been summarised in Figure 1. The results of the questionnaire were fed back to the Consultant Group Meeting in the BAOMS Annual Scientific Meeting in Birmingham, UK.

\section{7}

Activities in the second year included update of the web video resources on the BAOMS website to include some videos provided by the Reconstructive SSIG members for perforator/chimeric free flaps. A reconstructive SSIG cadaveric dissection and simulation study day entitled 'Minimising Morbidity if Maxillofacial Reconstruction' was organised in the University of Leeds, UK from 16-17 November 2017. The course was a combination of simulated teaching on midface models for planning and placement of zygomatic implants, harvest of the subscapular system of free flaps and familiarisation with patient specific planning systems with multiple industry representatives. The course was supported by several UK OMFS and an internationally renowned Consultant in Restorative Dentistry who first described the zygomatic implant perforated flap (1). Course delegates consisted of consultants and higher surgical trainees from OMFS and Plastic Surgery. 


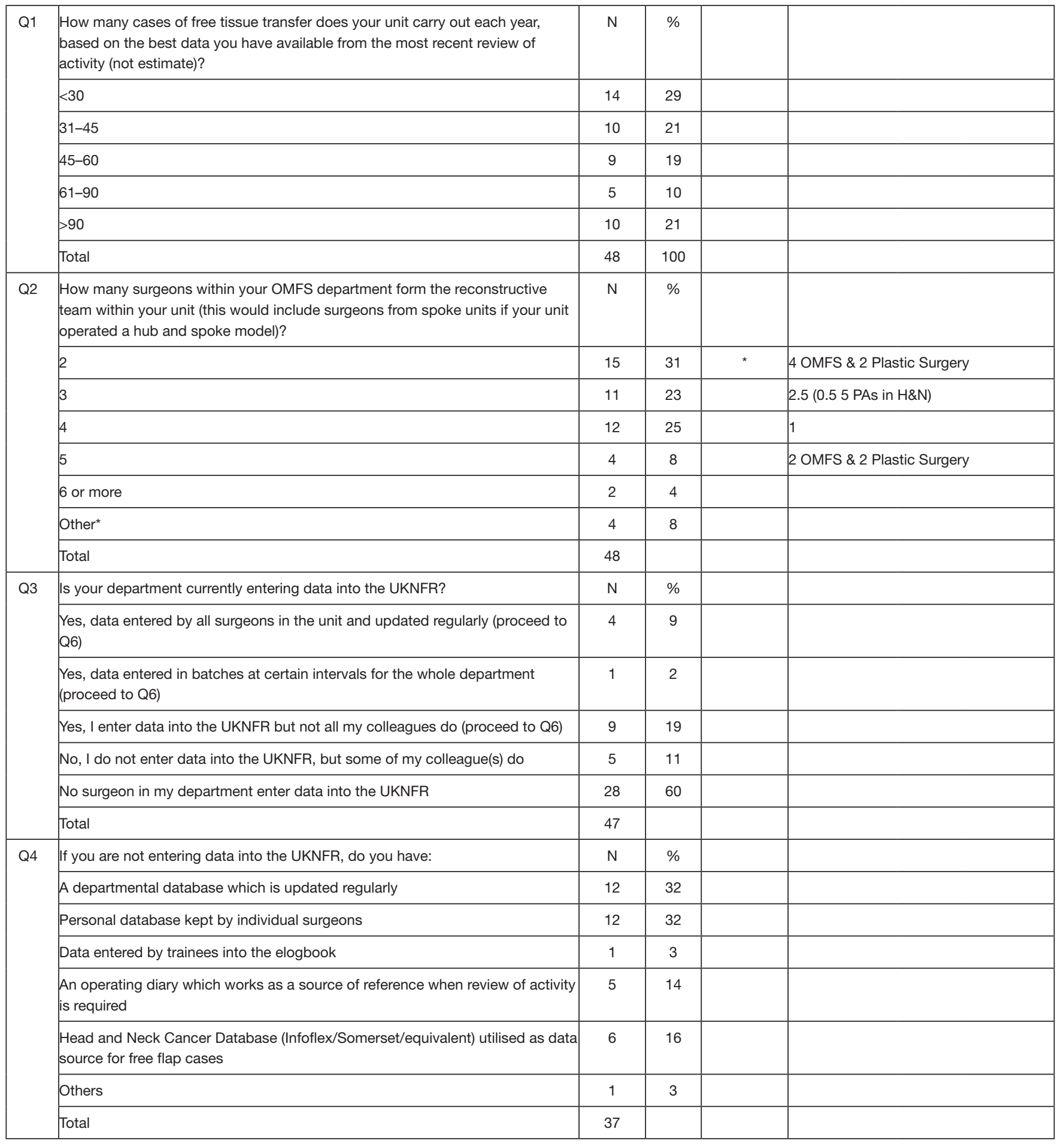




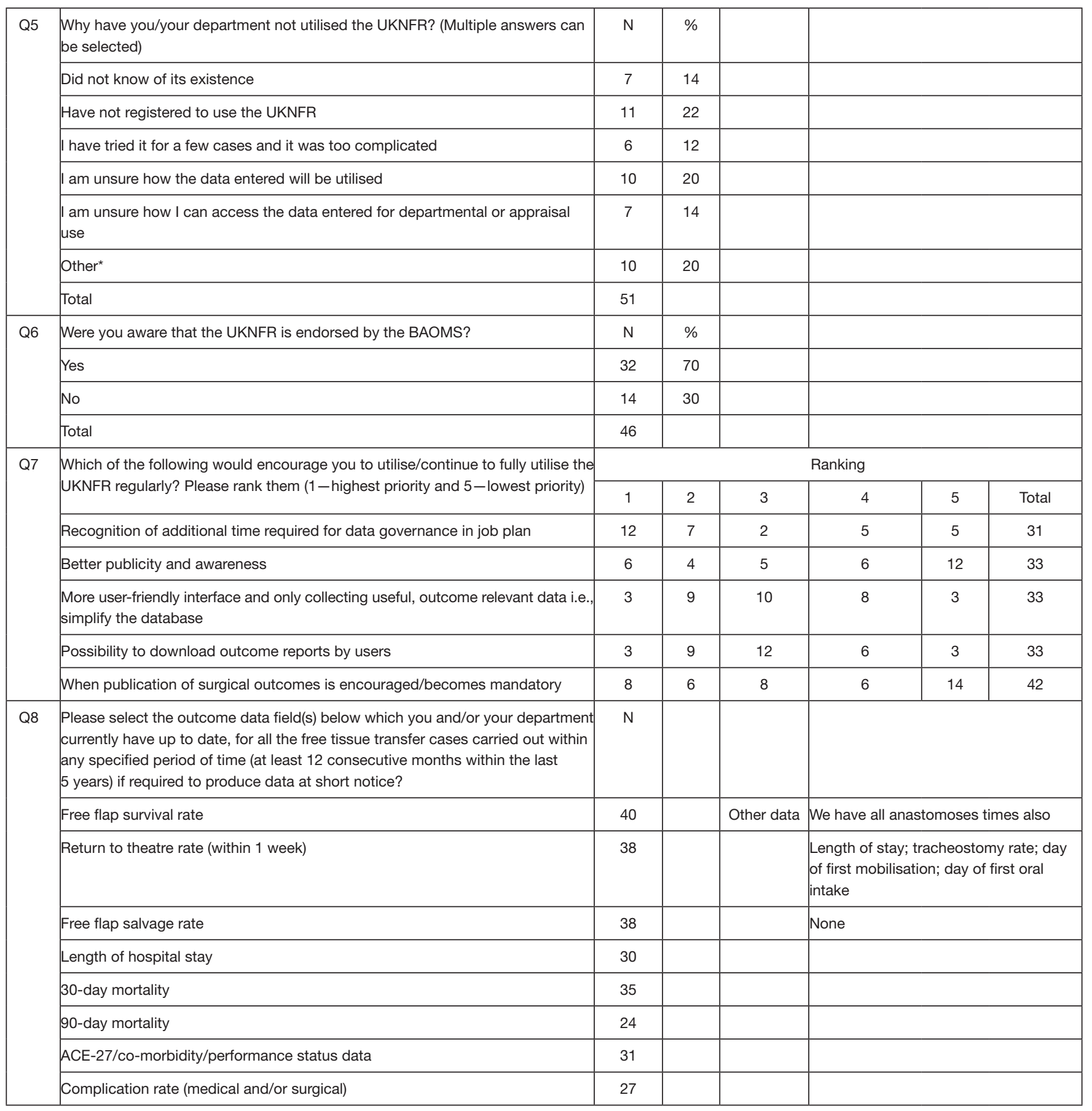




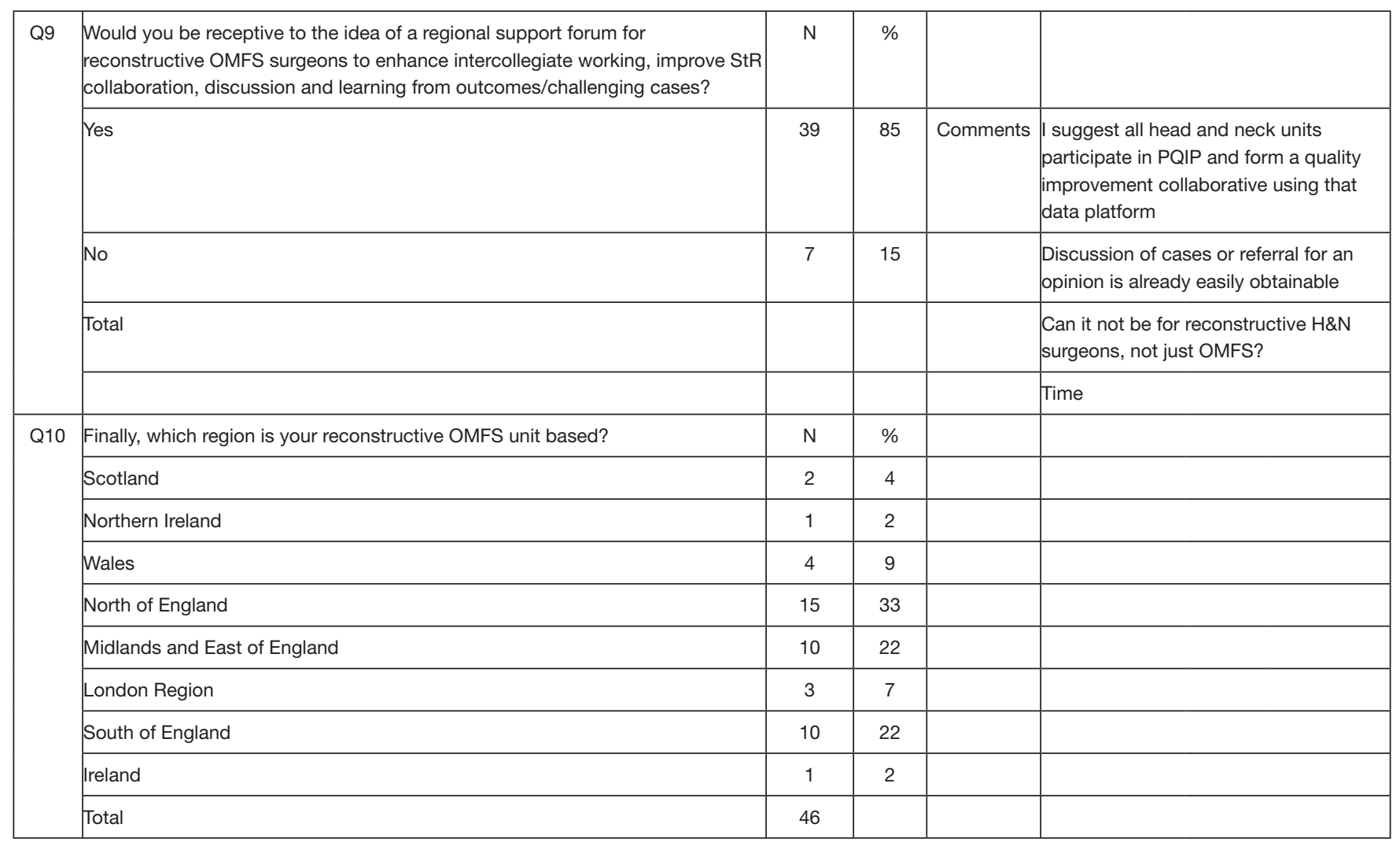

Figure 1 Questionnaire and responses ( $\mathrm{n}=48$ and percentages of responses indicated where relevant) from BAOMS Reconstruction SSIG fellows in 2016. BAOMS, the British Association of Oral and Maxillofacial Surgeons; SSIG, Sub-Specialty Interest Group; UKNFR, UK National Flap Registry; OMFS, Oral and Maxillofacial Surgeons; PAs, programmed activities unit (equivalent to 4 hours); H\&N, Head \& Neck; ACE-27, Adult Comorbidity Evaluation 27; PQIP, Perioperative Quality Improvement Project.

Initial discussions and planning with UK OMFS surgeons were held regarding the conception of a registry for the utilisation of patient specific osteosynthesis plates in composite mandibular reconstruction. Its use has been on the increase nationally, hence the development of a multi-centred database to monitor patient specific device safety and comparison of plate removal rates was essential to inform OMFS surgeons of clinical outcomes between different fixation systems: reconstruction plates $v$. miniplates, patient specific vs. stock plates.

The BAOMS Reconstructive SSIG section of the BAOMS website underwent reorganisation of content, layout and addition of new content which included upload of patient information leaflets on various types of flaps utilised in head and neck reconstruction (acknowledgements to the Aintree University Hospital Head and Neck Team).

\section{8}

The topic of invasive free flap monitoring was discussed quite frequently amongst UK OMFS teams this year and we were approached by an industry partner to collaborate on working towards a clinical consensus from the SSIG regarding the role of implantable Doppler monitoring in head and neck free flap reconstruction. An industry-clinician collaborative session on the role of implantable Doppler free flap monitoring in head and neck reconstructive surgery was held at BAOMS Annual Scientific Meeting in Durham, UK. The session was entitled, BAOMS Reconstruction SSIG: achieving a consensus for utilisation of implantable Doppler in maxillofacial reconstruction-collaboration between clinicians and industry. The session content was as follows: 
* Summary of evidence in the literature: implantable free flap monitoring;

* Rationale for implantable free flap monitoring in reconstructive OMFS;

* Rationale against implantable free flap monitoring in reconstructive OMFS;

* Utility of NHS OPCS Classification of Interventions and Procedures coding data to support the practice of reconstructive OMFS and support from industry in the implementation of implantable free flap monitoring.

It was concluded that invasive free flap monitoring should form part of the package of standard of care in head and neck reconstruction. Whether it should be utilised in all cases or in high-risk/stake patient cases (2), will depend on the experience and resources available within each respective OMFS unit. The prospective registry for patient specific mandibular osteosynthesis plate reconstruction was included as part of the BAOMS Quality and Outcomes in Oral and Maxillofacial Surgery (QOMS) registry activity.

The SSIG was asked by NHS England to provide clinical expertise in a consultation on the Ablative Surgery, MOld technique with afterloading brachytherapy and immediate surgical Reconstruction (AMORE), after chemotherapy, protocol for the management of paediatric rhabdomyosarcoma in the England and Wales.

The main activity of the SSIG for this year was involvement with the BAOMS QOMS in surgery quality improvement initiative (3). Escalating healthcare expenditure in the NHS is unsustainable, and service commissioners need to provide cost-effective surgical procedures that demonstrate clear health benefits in line with best evidence-based practice. Patients should expect, and demand, high quality care and the best possible surgical outcomes from OMFS, but the specialty lags other surgical specialties in publication of clinical outcomes across the UK. BAOMS, under its 2018 president Mr. Ian Martin, and with the support of council, initiated a specialty-wide quality improvement and clinical effectiveness programme, BAOMS QOMS. This reflected the president's view that implementing systematic quality improvement in OMFS and ensuring effectiveness of care provided based upon appropriate metrics were key to the continued successful development of surgical care in the NHS and reflected the core culture of the association.

\section{9}

Continued engagement with BAOMS QOMS in the development of the Reconstruction and Oncology audit questionnaires was the primary activity for this year. This aspect of SSIG took up the most part of the year for multiple meetings for consultation with the BAOMS QOMS working group, the National Consultant Improvement Project (NCIP) team (NHS Improvement) and representative from ENT-UK, BAPRAS and British Association of Head and Neck Oncology (BAHNO). The SSIG provided consultation to the NCIP team to locally validate centrally held hospital episode statistics (HES) data and refine the algorithms utilised to accurately capture data on various common head and neck oncology and reconstruction activity within the NHS in England and Wales $(4,5)$. The BAOMS QOMS pilot project started in December 2019. The SSIG was invited to co-author the first report of the UNKNFR, which was published in December 2019 (6).

\section{0}

The Reconstruction SSIG provided comments and consultation to the process of formulation of The UK Mucosal Melanoma Guidelines which was published this year (7). Due to the COVID-19 pandemic, the Reconstruction SSIG lead was invited to be a part of the Head and Neck Cancer Writing Group of the COVIDSurg Collaborative (8) and supported the BAOMS COVID projects. The BAOMS QOMS pilot project concluded at the end of March 2020, partly due to the COVID-19 pandemic. The lead and deputy lead of the SSIG demitted from office in July 2020.

\section{Discussion}

The BAOMS Reconstruction SSIG has been the most active SSIG in BAOMS during the 5-year period reported [2016-2020], its activities have provided continuing professional development for colleagues (clinical and industry partners) and Oral and Maxillofacial Surgical trainees, supported patient education and provision of information and engaged actively with BAOMS quality improvement initiatives. The SSIG has provided 
representation of the specialty and BAOMS in national high-profile publications such as the 2019 UKNFR report and UK Mucosal Melanoma Guidelines. Furthermore, it has supported the commissioners of clinical services, NHS England, in the provision of clinical expert opinion. Overall, the role of the Reconstruction SSIG has been pivotal in tackling important and topical issues in head and neck reconstruction in a timely manner for the specialty. This varied list of activities is something that could clearly develop in the future, according to the priorities of its members and leadership. The roles of SSIG lead and deputy lead, was highly rewarding for the authors. Despite the additional workload and responsibilities, the authors have been beneficiaries of increased network of collaboration with clinical and industry colleagues, strategically placed to develop various non-clinical skills such as leadership, managerial and higher-level communication with the healthcare provision infrastructure. The concept of a SSIG has been a successful innovation by BAOMS and the authors commend the BAOMS council for this idea. We would suggest that other specialty associations consider this model to allow areas of subspecialty interests to develop and flourish for the advancement of surgical practice and patient care.

\section{Acknowledgments}

Funding: None.

\section{Footnote}

Provenance and Peer Review: This article was commissioned by the editorial office, Frontiers of Oral and Maxillofacial Medicine, for the series "Head and Neck Reconstruction". The article has undergone external peer review.

Conflicts of Interest: Both authors have completed the ICMJE uniform disclosure form (available at https:// fomm.amegroups.com/article/view/10.21037/fomm-213/coif). The series "Head and Neck Reconstruction" was commissioned by the editorial office without any funding or sponsorship. MWSH served as the unpaid Guest Editor of the series, and serves as an unpaid editorial board member of Frontiers of Oral and Maxillofacial Medicine from October 2019 to September 2023. The author has no other conflicts of interest to declare.
Ethical Statement: The authors are accountable for all aspects of the work in ensuring that questions related to the accuracy or integrity of any part of the work are appropriately investigated and resolved.

Open Access Statement: This is an Open Access article distributed in accordance with the Creative Commons Attribution-NonCommercial-NoDerivs 4.0 International License (CC BY-NC-ND 4.0), which permits the noncommercial replication and distribution of the article with the strict proviso that no changes or edits are made and the original work is properly cited (including links to both the formal publication through the relevant DOI and the license). See: https://creativecommons.org/licenses/by-nc-nd/4.0/.

\section{References}

1. Butterworth CJ, Rogers SN. The zygomatic implant perforated (ZIP) flap: a new technique for combined surgical reconstruction and rapid fixed dental rehabilitation following low-level maxillectomy. Int J Implant Dent 2017;3:37.

2. Ho MW, Cassidy C, Brown JS, et al. Rationale for the use of the implantable Doppler probe based on 7 years' experience. Br J Oral Maxillofac Surg 2014;52:530-4.

3. McMahon J, Puglia F, Martin I, et al. Measuring healthrelated benefit and quality of care in oral and maxillofacial surgery: British Association of Oral and Maxillofacial Surgeons Outcomes Project. Br J Oral Maxillofac Surg 2018;56:439-43.

4. Nouraei SA, Middleton SE, Hudovsky A, et al. Role of reconstructive surgery in the management of head and neck cancer: a national outcomes analysis of 11,841 reconstructions. J Plast Reconstr Aesthet Surg 2015;68:469-78.

5. Nouraei SA, Mace AD, Middleton SE, et al. A stratified analysis of the perioperative outcome of 17623 patients undergoing major head and neck cancer surgery in England over 10 years: Towards an Informatics-based Outcomes Surveillance Framework. Clin Otolaryngol 2017;42:11-28.

6. Hazari A, Cole R, Fowler C, et al. First UK National Flap Registry Report 2019. 2019. Available online: http://www. bapras.org.uk/docs/default-source/default-documentlibrary/uknfr-first-report-4-dec-2019.pdf?sfvrsn=2http:// www.bapras.org.uk/docs/default-source/default- 
document-library/uknfr-first-report-4-dec-2019. pdf? sfvrsn=2

7. Mucosal Melanoma Guideline-Resources. 2020. Available online: https://melanomafocus.com/activities/mucosalguidelines/mucosal-melanoma-resources/

doi: $10.21037 /$ fomm-21-3

Cite this article as: Ho MWS, Nugent M. The role of a reconstruction subspecialty group in the national surgical specialty association: the journey, experiences and output for the advancement of head and neck reconstruction. Front Oral Maxillofac Med 2022;4:8.
8. COVIDSurg Collaborative. Head and neck cancer surgery during the COVID-19 pandemic: An international, multicenter, observational cohort study. Cancer 2021;127:2476-88. 